\title{
NOTE ON DEDUCED PROBABILITY DISTRIBUTIONS
}

\author{
R. VON MISES
}

In this Bulletin, December, 1936, A. H. Copeland* resumed the study of the problem first suggested by H. Poincaré: How can the fact of uniform probability distribution, which we meet so frequently in different games of chance, be explained? Recently E. Hopf devoted a profound essay $\dagger$ to this question and he has just published a short note $\ddagger$ dealing with his principal results. I want to contribute a quite simple remark which seems to show how far the results are independent of the particular form of dynamical equations.

We assume that there exists a density function $f(x)$ for the onedimensional variable $x$, such that $\int_{a}^{b} f(x) d x$ denotes the probability that the value of $x$ falls in the interval $(a, b)$ and $\int_{-\infty}^{\infty} f(x) d x=1$. If between $x$ and $y$ there is established a one-to-one correspondence

$$
y=y(x), \quad x=x(y),
$$

the given density function $f(x)$ leads to a new density function $g(y)$ defined by

$$
g(y)=f(x) \frac{d x}{d y} .
$$

The integral $\int_{a}^{b} g(y) d y$ gives, of course, the probability that $y$ belongs to the interval $(a, b)$ and $\int_{-\infty}^{\infty} g(y) d y=1$.

Now we suppose $y$ to be an "angular" variable, that is, instead of $y$ we consider the new variable:

$$
\eta=y-[y], \quad 0 \leqq \eta<1,
$$

and try to determine the probability distribution $\phi(\eta)$ of $\eta$. Evidently, if $\nu$ is a positive or negative integer, the probability density of $\eta$ is given by

(4) $\phi(\eta)=\sum_{\nu} g(\eta+\nu)=\sum_{\nu} f\left(x_{\nu}\right)\left(\frac{d x}{d y}\right)_{x=x_{\nu}} ; \quad x_{\nu}=x(\eta+\nu)$.

* Vol. 42, p. 895 .

$\dagger$ Journal of Mathematics and Physics, Massachusetts Institute of Technology, vol. 13 (1934).

¥Jahresbericht der Deutschen Mathematiker-Vereinigung, vol. 46 (1936), p. 179. 
We may suppose the transformation ratio $d x / d y>0$. In this case the consecutive values $\cdots x(-2), x(-1), x(0), x(1), x(2), \cdots$ define an infinite set of intervals corresponding to intervals of length 1 on the $y$-axis. Let $\eta^{\prime}$ and $\eta^{\prime \prime}$ be two values of $\eta$; then the corresponding values $x_{\nu}^{\prime}=x\left(\eta^{\prime}+\nu\right)$ and $x_{\nu}^{\prime \prime}=x\left(\eta^{\prime \prime}+\nu\right)$ fall in the same interval $(x(\nu), x(\nu+1))$. Therefore, the difference between the values of the products

$$
f\left(x_{\nu}^{\prime}\right)\left(\frac{d x}{d y}\right)_{x=x_{\nu}^{\prime}} \quad \text { and } \quad f\left(x_{\nu}^{\prime \prime}\right)\left(\frac{d x}{d y}\right)_{x=x_{\nu}^{\prime \prime}}
$$

is less than or equal to the variation of the product $f \cdot d x / d y$ through the interval $(x(\nu), x(\nu+1))$, and the difference between the two values of the sum (4) for $\eta^{\prime}$ and $\eta^{\prime \prime}$ does not exceed the value of the total variation of the same product. Hence, our theorem follows:

The maximum difference between two values of the deduced probability density $\phi(\eta)$ is less than or equal to the total variation of the product of initial density $f(x)$ by the transformation ratio $d x / d y$.

If we consider an infinite set of similar problems where the initial distribution $f(x)$ remains unchanged and the transformation ratio is multiplied by a parameter $\lambda$, then the deduced distribution $\phi(\eta)$ approaches uniformity as the parameter $\lambda$ approaches 0 and the functions $f(x)$ and $d x / d y$ are of finite variation.

The mechanical example mentioned by Copeland and by Hopf consists in a system rotating about a vertical axis and subjected to friction forces which depend on the instantaneous angular velocity $\omega$. The dynamical equation is given by

$$
\frac{d \omega}{d t}=-r(\omega)
$$

Let $x$ be the initial value $\omega_{0}$ of $\omega$. Until the system comes to rest, a point at the distance 1 from the axis will travel a distance which may be designed by $2 \pi y$. Then it follows from (5) that

$$
2 \pi y=\int \omega d t=\int_{0}^{x} \frac{\omega d \omega}{r(\omega)}, \quad \frac{d x}{d y}=2 \pi \frac{r(x)}{x} .
$$

Copeland supposes the friction $r(x)$ to be proportional to a parameter $\lambda$, but the distribution $f(x)$ to be independent of $\lambda$. In this case it is clear that, in consequence of $(6), f(x) \cdot d x / d y$ approaches zero with $\lambda \rightarrow 0$, and our theorem shows that the asymptotic value of $\phi(\eta)$ is a constant. 
On the other hand, Hopf considers the initial distribution to be given in the form $f(x)=f_{1}(x-\lambda)$, where $f_{1}$ is a function of one variable and $\lambda$ a parameter. Moreover, he supposes that

$$
\lim _{x \rightarrow \infty} \frac{r(x)}{x}=0 \text {. }
$$

We find $f(x) \cdot d x / d y=2 \pi f_{1}(x-\lambda) \cdot r(x) / x=2 \pi f_{1}(z) r(\lambda+z) /(\lambda+z)$, which approaches zero, according to (7), as $\lambda$ increases. If the functions $f_{1}$ and $r / x$ are of finite variation, it follows from our theorem that $\phi(\eta)$ approaches uniformity.

It is a quite different question to decide whether the foregoing investigation is or is not sufficient to explain the fact of the nearly perfect uniformity of distribution in a particular case of a real game. Let us consider a sort of roulette consisting of a billiard ball which runs in a smooth circular channel subjected to a constant resistance $r(\omega)=c$; the number of revolutions is found to vary from about 8.1 to 12.1 . Our equation (6) gives

$$
2 \pi y=\frac{x^{2}}{2 c}, \quad \frac{d x}{d y}=\frac{2 \pi c}{x}=\left(\frac{\pi c}{y}\right)^{1 / 2}, \quad x=2(\pi c y)^{1 / 2} .
$$

Therefore $x$ varies from $9(0.4 \pi c)^{1 / 2}$ to $11(0.4 \pi c)^{1 / 2}$, and if we assume $f(x)$ to be constant in this interval of length $2(0.4 \pi c)^{1 / 2}$, we find

$$
g(y)=\frac{1}{2(0.4 \pi c)^{1 / 2}}\left(\frac{\pi c}{y}\right)^{1 / 2}=\frac{1}{2(0.4 y)^{1 / 2}}, \quad 8.1 \leqq y \leqq 12.1 .
$$

The resulting density function $\phi(\eta)$ is a monotonic decreasing function in the interval from $\eta=0.1$ to $\eta=1.1$. If we divide the whole circle in two parts from $\eta=0.1$ to $\eta=0.6$ and from 0.6 to 1.1 , it follows that the probability of a rest position in the first of these semicircles is

$$
\int_{8.1}^{8.6} g(y) d y+\int_{9.1}^{9.6} \cdots+\int_{10.1}^{10.6} \cdots+\int_{11.1}^{11.6} \cdots=0.506 .
$$

The excess of $1.2 \%$ is doubtless too large for a fair game of chance. It seems that in such cases other circumstances increase the tendency towards uniformity.

UNIVERSITY OF ISTANBUL 